\title{
Long range corrections for computer simulations of adsorption
}

\author{
F. SIPERSTEIN ${ }^{1}$, A. L. MYERS ${ }^{1 *}$ and O. TALU ${ }^{2}$ \\ ${ }^{1}$ Department of Chemical Engineering, University of Pennsylvania, Philadelphia, \\ PA 19104, USA \\ ${ }^{2}$ Chemical Engineering Department, Cleveland State University, Cleveland, \\ OH 44115, USA
}

(Received 7 August 2001; accepted 18 August 2001)

\begin{abstract}
Long range corrections are routinely applied to simulations of bulk fluids by assuming that the radial distribution function is unity beyond a certain cutoff radius for pairwise interactions. Similar long range corrections for gas-solid interactions in adsorption frequently are ignored because of the anisotropic structure of the solid. However, the error associated with assuming isotropy beyond the cutoff radius is small compared with the magnitude of the long range correction. The long range correction to the Henry constant for a cutoff radius of $13 \AA$ is $14 \%$ for $\mathrm{CH}_{4}$ and $70 \%$ for $\mathrm{SF}_{6}$ for adsorption in silicalite at $298 \mathrm{~K}$. The large errors incurred by neglecting long range corrections can be concealed by increasing the well depth of the gassolid interaction, but this approximation reduces the accuracy and portability of the potential parameters. Consistency in the cutoff radius is more important than the inclusion or neglect of long range corrections to the energy.
\end{abstract}

\section{Introduction}

Molecular simulation of adsorption is based upon a detailed model of the potential energy of the adsorbate molecules as a function of their location and orientation inside the pores of the solid adsorbent. Assuming pairwise additivity, the total potential energy of a configuration is obtained by summing gas-gas and gas-solid interaction energies. At the limit of zero loading, the gas-gas interactions are negligible and the energy consists entirely of gas-solid interactions. The total potential energy of a gas molecule adsorbed at a particular location includes interactions between the gas molecule and every other atom in the simulation box, as well as images in the surrounding boxes. This summation contains an infinite number of terms and is impossible to calculate in practice [1].

The approximation for dispersion/repulsion forces in bulk fluids is to use a spherical cutoff radius $\left(r_{\mathrm{c}}\right)$. Corrections for the missing long range part of the potential are based on the approximation that the radial distribution function $g(r) \approx 1$ for $r>r_{\mathrm{c}}$. Long range corrections can be introduced at the end of the simulation in ensembles where the density is constant. In NPT and $\mu V T$ ensembles, the long range corrections must be applied to the instantaneous energies and pressures during the course of the simulation, because the corrections change as the density fluctuates [2].

\footnotetext{
* Author for correspondence. e-mail: amyers@seas.upenn.
}

In adsorption simulations it is necessary to distinguish between two types of long range correction: one for the fluid-fluid interactions and another for the fluid-solid interactions. Fluid-fluid corrections for adsorption of spherical molecules on a flat infinite solid surface were studied by Rowley et al. [2], who showed that long range corrections are necessary only for high coverage (more than two monolayers). This observation implies that long range corrections for fluid-fluid interactions are negligible in microporous adsorbents for which the pore size is not much larger than the adsorbed molecule. Based upon the relative densities of solid and fluid atoms in adsorbents, long range corrections for fluidfluid interactions are at least an order of magnitude smaller than solid-fluid tail corrections. Therefore only fluid-solid interactions are considered here.

Fluid-solid corrections are sometimes ignored and sometimes included by assuming a homogenous density for the solid beyond the cutoff radius [3, 4]. The purpose of this paper is to study the importance of long range corrections for adsorption of gases in microporous adsorbents.

\section{Long range corrections for bulk fluids}

Assuming pairwise additivity, the potential energy $\phi$ in a system of density $\rho$ is [5]

$$
\phi=\frac{1}{2} \rho \int_{0}^{\infty} \phi(r) g(r) 4 \pi r^{2} \mathrm{~d} r,
$$


where $\rho$ is the molecular density, $\phi(r)$ is the pair potential, and $g(r)$ is the radial distribution function. Computer simulations frequently use pair potentials with a spherical cutoff at $r_{\mathrm{c}}$, in which case the simulation results may be corrected for the missing long range part of the potential by assuming that $g(r)=1$ for $r>r_{\mathrm{c}}$. Specifically, for the Lennard-Jones 12-6 pair potential,

$$
\phi(r)=4 \epsilon\left[\left(\frac{\sigma}{r}\right)^{12}-\left(\frac{\sigma}{r}\right)^{6}\right] .
$$

Insertion of equation (2) into (1) gives the long range correction for the molecular potential energy

$$
\phi_{\text {Irc }}=\frac{1}{2} \rho \int_{r_{\mathrm{c}}}^{\infty} \phi(r) 4 \pi r^{2} \mathrm{~d} r=\frac{8}{3} \pi \rho \epsilon \sigma^{3}\left[\frac{1}{3}\left(\frac{\sigma}{r_{\mathrm{c}}}\right)^{9}-\left(\frac{\sigma}{r_{\mathrm{c}}}\right)^{3}\right]
$$

\section{Long range corrections for gas-solid interactions}

The gas-solid potential energy of an adsorbate molecule, which is a function of its position $\mathbf{r}$, is obtained by summing over the atoms of the solid adsorbent

$$
\phi(\mathbf{r})=\sum_{j} \phi\left(r_{1 j}\right),
$$

where $r_{1 j}$ is the distance between an adsorbate molecule located at $\mathbf{r}$ and atom $j$ of the adsorbent located at $\mathbf{r}_{j}$

$$
r_{1 j}=\left|\mathbf{r}-\mathbf{r}_{j}\right| \text {. }
$$

The summation assumes that the energies are pairwise additive and that the adsorbate molecule is spherical, so that its energy is independent of orientation. In practice, the summation of equation (4) is limited to a cutoff radius $r_{\mathrm{c}}$ such that

$$
\phi(\mathbf{r})=\phi_{\mathrm{c}}(\mathbf{r})+\phi_{\mathrm{lrc}},
$$

where $\phi_{\mathrm{c}}(\mathbf{r})$ is for the potential truncated at $r=r_{\mathrm{c}}$

$$
\phi_{\mathrm{c}}(\mathbf{r})=\sum_{r_{1 j}=0}^{r_{\mathrm{c}}} \phi\left(r_{1 j}\right)
$$

and the long range correction to the energy of an adsorbate molecule is estimated by the approximation $g(r)=1$, similar to equation (3) but without the factor of $\frac{1}{2}$ that corrects for duplicate counting of pairwise interactions in bulk fluids

$$
\phi_{\text {Irc }}=\rho_{\mathrm{s}} \int_{r_{\mathrm{c}}}^{\infty} \phi(r) 4 \pi r^{2} \mathrm{~d} r=\frac{16}{3} \pi \rho_{\mathrm{s}} \epsilon \sigma^{3}\left[\frac{1}{3}\left(\frac{\sigma}{r_{\mathrm{c}}}\right)^{9}-\left(\frac{\sigma}{r_{\mathrm{c}}}\right)^{3}\right] \text {, }
$$

where $\rho_{\mathrm{S}}$ is the density of solid atoms. In siliceous materials like silicalite, the less accessible silicon atoms frequently are ignored, and the density $\rho_{\mathrm{s}}$ refers only to oxygen atoms.

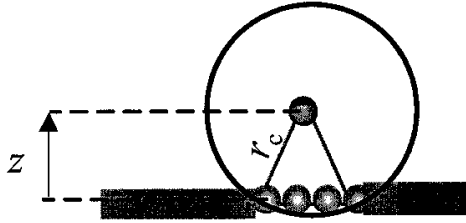

Figure 1. Spherical cutoff radius for adsorption on a flat surface.

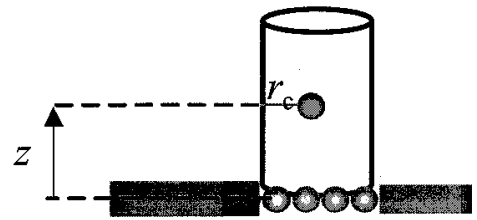

Figure 2. Cylindrical cutoff radius for adsorption on a flat surface.

\subsection{Special case of flat surfaces}

Adsorption potentials for Lennard-Jones spheres on smooth infinite surfaces are well known [6, 7]; these smoothed potentials obviously require no long range corrections. However, molecular simulations of adsorption on structured walls require a cutoff radius and long range corrections. For a single plane, a pseudo-spherical cutoff radius (see figure 1) can be applied by assuming that the surface is smooth for $r>r_{\mathrm{c}}$. The long range correction for the Lennard-Jones 12-6 potential is then given by

$$
\begin{array}{ll}
\phi_{\text {lrc }}=4 \pi \rho_{\mathrm{s}} \epsilon \sigma^{2}\left[\frac{1}{5}\left(\frac{\sigma}{z}\right)^{10}-\frac{1}{2}\left(\frac{\sigma}{z}\right)^{4}\right] & \text { for } z>r_{\mathrm{c}}, \\
\phi_{\mathrm{lrc}}=4 \pi \rho_{\mathrm{s}} \epsilon \sigma^{2}\left[\frac{1}{5}\left(\frac{\sigma}{r_{\mathrm{c}}}\right)^{10}-\frac{1}{2}\left(\frac{\sigma}{r_{\mathrm{c}}}\right)^{4}\right] & \text { for } z<r_{\mathrm{c}}
\end{array}
$$

where $\rho_{\mathrm{s}}$ is the area density of solid atoms in the plane. Alternatively, the long range correction for a single plane with a cylindrical cutoff radius (see figure 2) is

$$
\phi_{\text {Irc }}=4 \pi \rho_{\mathrm{s}} \epsilon \sigma^{2}\left[\frac{1}{5}\left(\frac{\sigma^{10}}{\left(z^{2}+r_{\mathrm{c}}^{2}\right)^{5}}\right)-\frac{1}{2}\left(\frac{\sigma^{4}}{\left(z^{2}+r_{\mathrm{c}}^{2}\right)^{2}}\right)\right] .
$$

Long range corrections can be applied to walls or slits formed from stacks of infinite parallel planes by summation of the above corrections for single planes.

\section{Effect of long range corrections upon the Henry constant}

The values of gas-solid potential parameters extracted from the Henry constants of experimental adsorption isotherms are strongly affected by long range corrections. The initial slope of an adsorption isotherm is called the Henry constant $(B / k T)$, 


$$
\lim _{P \rightarrow 0} \frac{\mathrm{d} N}{\mathrm{~d} P}=\frac{B}{k T},
$$

where $N$ is absolute amount adsorbed per unit mass of adsorbent and $B$ is the absolute adsorption second virial coefficient

$$
B=\frac{1}{m} \int \mathrm{e}^{-\beta \phi(\mathbf{r})} \mathrm{d} \mathbf{r},
$$

where $\beta=1 /(k T)$. The excess adsorption second virial coefficient [6] has an integrand of $\left[\mathrm{e}^{-\beta \phi(\mathbf{r})}-1\right]$ and the integral is over the space occupied by the adsorbate molecules; the integral of equation (13) for the absolute value of $B$ is over a representative mass $m$ of the solid adsorbent and the exponential vanishes within the solid where $\phi \rightarrow \infty$. Insertion of equation (6) in (13) yields

$$
B=\frac{\mathrm{e}^{-\beta \phi_{\mathrm{Irc}}}}{m} \int \mathrm{e}^{-\beta \phi_{\mathrm{c}}(\mathbf{r})} \mathrm{d} \mathbf{r} .
$$

The exponential character of the long range correction ( $\mathrm{e}^{-\beta \phi_{\text {Irc }}}$ ) makes $B$ highly sensitive to the size of the cutoff radius. Since $\phi_{\operatorname{lrc}}$ is negative, $B$ is underestimated if the long range correction is ignored.

The temperature dependence of $B$ is related to the differential enthalpy of adsorption. The absolute differential enthalpy at the limit of zero loading (negligible gas-gas interactions) is given by [8]

$$
\bar{h}=\frac{\int \phi(\mathbf{r}) \mathrm{e}^{-\beta \phi(\mathbf{r})} \mathrm{d} \mathbf{r}}{\int \mathrm{e}^{-\beta \phi(\mathbf{r})} \mathrm{d} \mathbf{r}}-k T .
$$

Substitution of equation (6) into (15) yields

$$
\bar{h}=\frac{\int \phi_{\mathrm{c}}(\mathbf{r}) \mathrm{e}^{-\beta \phi_{\mathrm{c}}(\mathbf{r})} \mathrm{d} \mathbf{r}}{\int \mathrm{e}^{-\beta \phi_{\mathrm{c}}(\mathbf{r})} \mathrm{d} \mathbf{r}}+\phi_{1 \mathrm{rc}}-k T .
$$

Alternatively, equation (16) may be derived from equation (14) using the thermodynamic relationship [9]

$$
\bar{h}=-\frac{d \ln B}{\mathrm{~d} \beta}-k T .
$$

The differential enthalpy $\bar{h}$ is exothermic and negative in sign; the positive value of the differential enthalpy is called the isosteric heat $\left(q_{s t}=-\bar{h}\right)$.

\section{Adsorption in silicalite}

The effect of long range corrections upon the values of the Henry constant and differential enthalpy is illustrated for the adsorption of non-polar gases in silicalite. Adsorption of alkanes in silicalite has been studied widely [3, 4, 10-23]. A cutoff radius $r_{\mathrm{c}}=13 \AA$ corresponding to the length of one side of the unit cell has been used frequently. More conservative calculations use cutoff radii of $19 \AA$ [21] or $20 \AA$ [22]. In one simulation [23], a cutoff radius of $8 \AA$ was used to speed up the calculation.

Silicalite is the siliceous form of ZSM-5 type materials with an MFI framework having a unit cell composition of $\mathrm{Si}_{96} \mathrm{O}_{192}$ and unit cell dimensions of 20.0, 19.9, and 13.4 A. A pre-tabulation scheme was utilized to calculate gas-solid potentials using crystallographic data [24] and a $0.01 \mathrm{~nm} 3$-dimensional grid. Grids were generated using cutoff radii of $1.0,1.3,2.0$, and $4.0 \mathrm{~nm}$. Only oxygen-adsorbate interactions were considered, and it was assumed that the presence of silicon atoms can be ignored. This assumption is justified because the silicon atoms are located at the centres of the $\mathrm{SiO}_{4}$ tetrahedra and are not in direct contact with the adsorbed molecules. The pre-tabulated grid was calculated in oneeighth of the unit cell and the solid-fluid interactions at any point in the unit cell were calculated from the Pnma symmetry of the MFI structure. Only 200000 of the 600000 nodes in the grid did not overlap with the oxygen atoms and thus were available as adsorption sites.

The calculation of the adsorption second virial coefficient and differential enthalpy was reduced to a summation over the pre-tabulation, which is very efficient compared with a Monte Carlo simulation. If $\mathcal{N}$ is the number of nodes in the pre-tabulation and $\phi_{j}$ is the energy at node $j$, then the expression for $B$ equivalent to equation (13) is

$$
B=\frac{1}{\mathcal{N} m} \sum_{j=1}^{\mathcal{N}} \mathrm{e}^{-\beta \phi_{j}}
$$

The summation equivalent of equation (15) for the differential enthalpy is

$$
\bar{h}=\frac{\sum_{j=1}^{\mathcal{N}} \phi_{j} \mathrm{e}^{-\beta \phi_{j}}}{\sum_{j=1}^{\mathcal{N}} \mathrm{e}^{-\beta \phi_{j}}}-k T .
$$

Lennard-Jones potential parameters used to describe adsorbate-oxygen interactions are given in table 1. These values taken from the literature [15] are not necessarily consistent with other reported values, but are adequate for estimating the magnitude of the long range corrections.

The density of oxygen atoms in silicalite is given by the number of oxygen atoms (192) in the unit cell [24]

$$
\rho_{\mathrm{s}}=\frac{192}{20.1 \times 19.9 \times 13.4}=0.0358 \text { atoms per } \AA^{3} \text {. }
$$

We calculated the methane-oxygen radial distribution function shown in figure 3 using the potential par- 


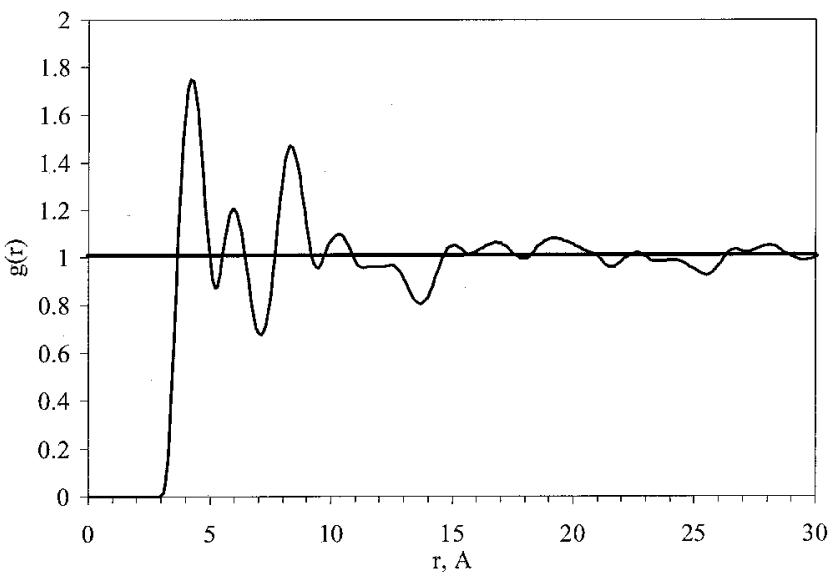

Figure 3. Gas-solid radial distribution function for adsorption of methane in silicalite at $100 \mathrm{kPa}$ and $300 \mathrm{~K}$.

Table 1. Lennard-Jones gas-solid potential parameters.

\begin{tabular}{lcc}
\hline Gas & $\epsilon k^{-1} / \mathrm{K}$ & $\sigma / \AA$ \\
\hline $\mathrm{CH}_{4}$ & 133.3 & 3.21 \\
$\mathrm{SF}_{6}$ & 147.2 & 3.97 \\
$\mathrm{CH}_{3}$ & 80.0 & 3.60 \\
$\mathrm{CH}_{2}$ & 58.0 & 3.60 \\
\hline
\end{tabular}

ameters in table 1 and methods described elsewhere [22]. The complicated structure of the radial distribution function is a consequence of the anisotropic structure of silicalite, which consists of intersecting straight and zig-zag channels. The structure in the radial distribution function persists strongly up to about $10 \AA$ and then converges slowly to unity in the range from $10 \AA$ to $30 \AA$.

Figure 4 shows the error in the Henry constant as a function of the cutoff radius without any long range corrections. Clearly the error associated with a cutoff radius of $10 \AA$ is unacceptable: a $23 \%$ error for $\mathrm{CH}_{4}$ and a $67 \%$ error for $\mathrm{SF}_{6}$. A cutoff radius of about $40 \AA$ is necessary to reduce the error in the Henry constant to $2 \%$.

Figure 5 shows the error in the differential enthalpy as a function of the cutoff radius without any long range corrections. The error associated with a cutoff radius of $13 \AA$ is $1.7 \%$ for $\mathrm{CH}_{4}$ and $3.2 \%$ for $\mathrm{SF}_{6}$. This relatively small error for the enthalpy seems inconsistent with the very large error in the Henry constant. However, the energy (enthalpy) of adsorption of $\mathrm{SF}_{6}$ is about $39 \mathrm{~kJ} \mathrm{~mol}^{-1}$, so an error of $3.2 \%$ in the energy is $1.25 \mathrm{~kJ}$ $\mathrm{mol}^{-1}$. According to equation (14), an error of $1.25 \mathrm{~kJ}$ $\mathrm{mol}^{-1}$ in the energy at $300 \mathrm{~K}$ generates a correction factor of $\exp \{(1250) /[(8.3145)(300)]\}=1.65$ for the Henry constant.

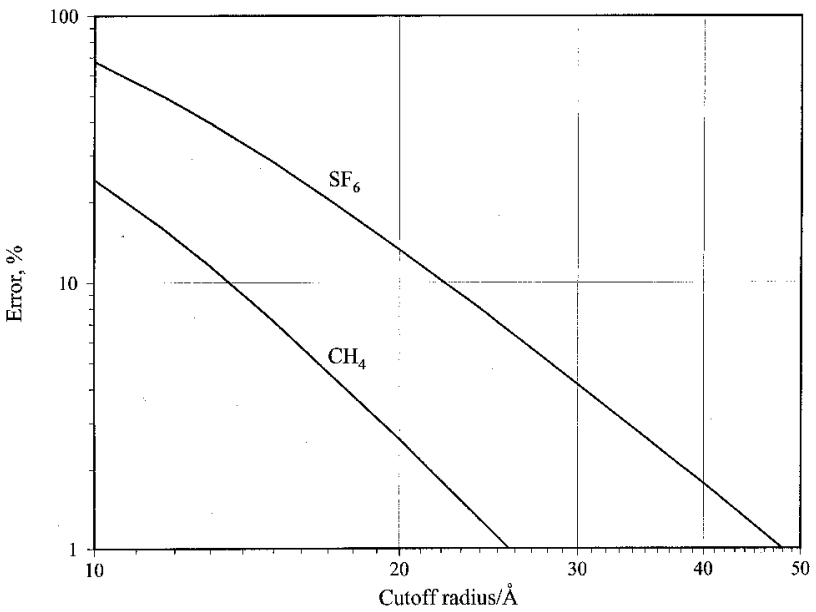

Figure 4. Error in the Henry constant for $\mathrm{SF}_{6}$ and $\mathrm{CH}_{4}$ adsorbed in silicalite at $300 \mathrm{~K}$ as a function of cutoff radius of gas-solid potential.

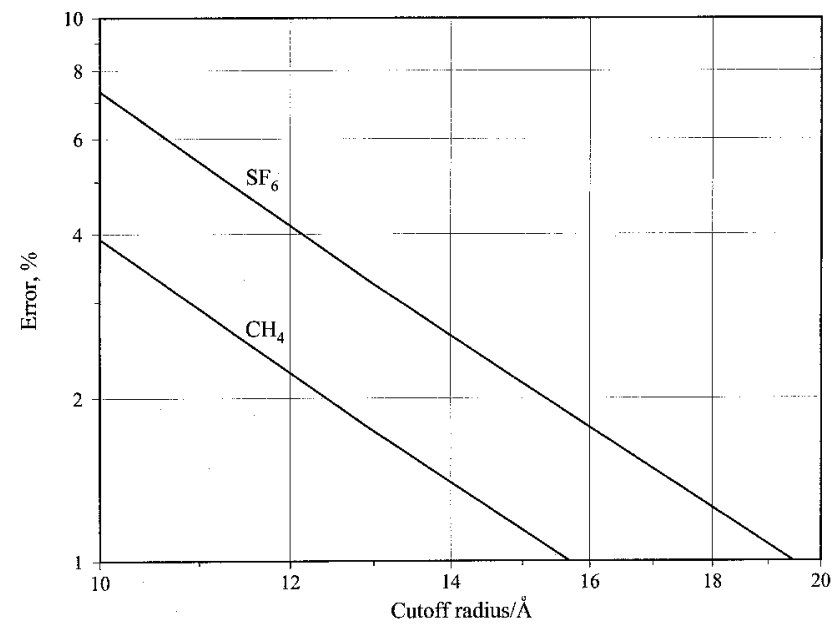

Figure 5. Error in the differential enthalpy for $\mathrm{SF}_{6}$ and $\mathrm{CH}_{4}$ adsorbed in silicalite at $300 \mathrm{~K}$ as a function of cutoff radius of gas-solid potential.

Figure 6 shows the error in the Henry constant for a cutoff radius of $13 \AA$ without any long range corrections in the case of adsorption of alkanes where the united atom approximation is used for each $\mathrm{CH}_{3}$ or $\mathrm{CH}_{2}$ group. The gas-solid interaction parameters were taken from table 1 . The long range correction is proportional to the number of beads in the alkane chain: the error of $10 \%$ for methane increases to an error of about $80 \%$ for n-pentane.

\section{Portability of potential parameters}

The sensitivity of the Henry constant to the cutoff radius raises the important issue of portability of potential parameters. The potential parameters in table 2 were derived from experimental data [25]. Note that these 


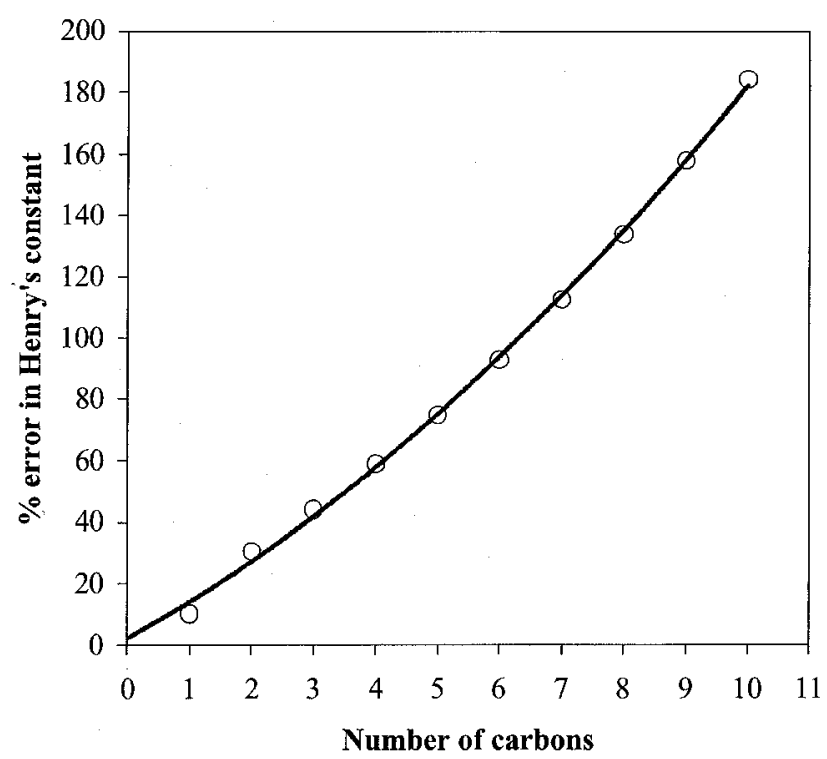

Figure 6. Error in the Henry constant for adsorption of alkanes in silicalite for a cutoff radius of $13 \AA$.

Table 2. Lennard-Jones gas-solid potential parameters derived for adsorption in silicalite for different cutoff radii.

\begin{tabular}{lcccc}
\hline & & & \multicolumn{2}{c}{$\epsilon \mathrm{k}^{-1} / \mathrm{K}$} \\
\cline { 4 - 5 } Gas & $\sigma / \AA$ & $r_{\mathrm{c}} / \AA$ & With LRC & Without LRC \\
\hline $\mathrm{CH}_{4}$ & 3.502 & 13 & 103.7 & 106.4 \\
$\mathrm{CH}_{4}$ & 3.502 & 20 & 103.8 & 104.5 \\
$\mathrm{CH}_{4}$ & 3.502 & 40 & 103.8 & 103.9 \\
$\mathrm{SF}_{6}$ & 4.085 & 13 & 117.3 & 122.3 \\
$\mathrm{SF}_{6}$ & 4.085 & 20 & 117.4 & 118.7 \\
$\mathrm{SF}_{6}$ & 4.085 & 40 & 117.4 & 117.6 \\
\hline
\end{tabular}

parameters differ slightly from values reported by other investigators. The collision diameter was held constant while adjusting the well depth $\epsilon$ to fit experiment for different cutoff radii, as shown in figure 7 . The open symbols were obtained by applying the long range exponential correction in equation (14) and the closed symbols were obtained by ignoring the long range correction. As observed previously, long range corrections become negligible at a cutoff radius of about $40 \AA$. For smaller cutoff radii, neglect of long range corrections requires a compensatory increase in the gas-solid well depth $\epsilon$.

The portability of potential parameters and their sensitivity to long range corrections was tested by predicting adsorption isotherms for $\mathrm{CH}_{4}$ and $\mathrm{SF}_{6}$ in siliceous faujasite using the potential parameters derived for silicalite. Siliceous faujasite has a cubic unit cell $\left(24.26 \AA\right.$ ) with a composition of $\mathrm{Si}_{192} \mathrm{O}_{384}$ [26]. The

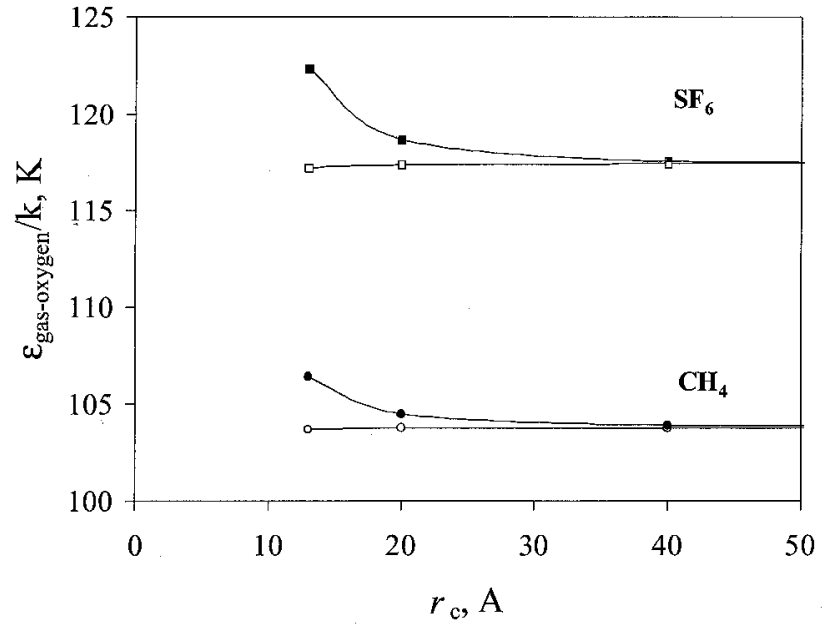

Figure 7. Lennard-Jones parameters for adsorption of $\mathrm{CH}_{4}$ and $\mathrm{SF}_{6}$ adsorbed in silicalite at $300 \mathrm{~K}$ for different cutoff radii. Open symbols include tail corrections; solid symbols calculated without tail corrections.

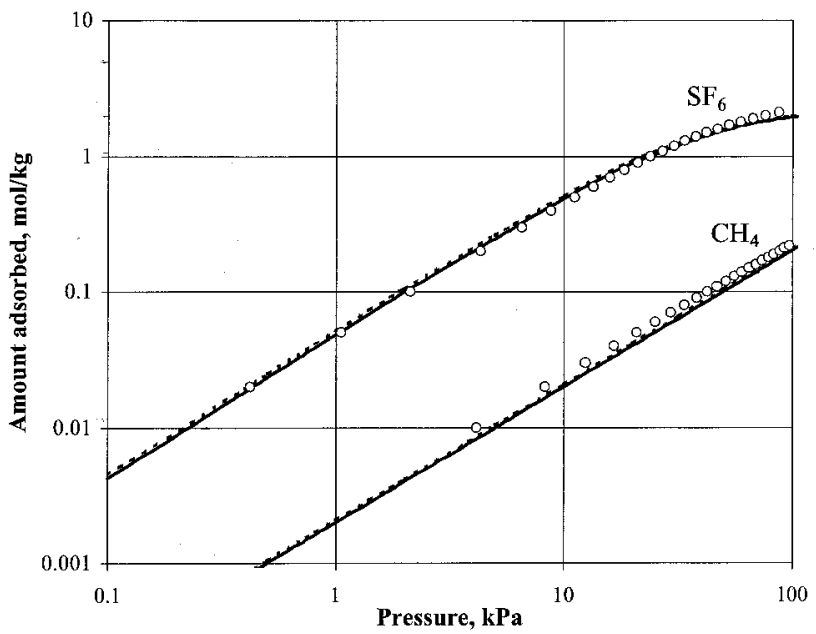

Figure 8. Test of portability of potential parameters for $\mathrm{SF}_{6}$ and $\mathrm{CH}_{4}$ adsorption on siliceous faujasite at $300 \mathrm{~K}$. Calculations based upon parameters derived in table 2 for silicalite with $r_{\mathrm{c}}=13 \AA$. The solid lines ignore long range corrections and the dashed lines include long range corrections, but the parameter $\epsilon$ for the solid line is larger, to adjust for the neglect of long range interactions. Symbols are experimental data [27].

potential energy was pre-tabulated for $1 / 16$ th of a unit cell using a cutoff radius of $13 \AA$. The energy at any point in the unit cell was obtained using the symmetry operations of the Fd-3m space group. The simulation box size was adjusted to contain no less than 10 adsorbed molecules at any time. For most of the simulations, simulation boxes containing $2 \times 2 \times 2$ or $3 \times 3 \times 3$ unit cells were used. The system was allowed to equilibrate for the first 1 million configurations of each run and averages were taken over the following 5 million configurations. 
Adsorption isotherms calculated for siliceous faujasite using the potential parameters derived for silicalite in table 2 are compared with experiment in figure 8 . The long range corrections were calculated from equation (8) using the density of oxygen atoms in faujasite, which is $72 \%$ of the density of oxygen atoms in silicalite. The dashed lines include long range corrections by equation (8); the solid lines compensate for neglect of long range corrections by using larger gas-solid energy parameters $\epsilon$ given in table 2. Since the difference between the calculated isotherms is about $5 \%$, the portability of gas-solid potentials is affected significantly by the neglect of long range corrections, even if the cutoff radius is fixed.

\section{Conclusion}

The adsorption isotherm is extremely sensitive to the cutoff radius used for gas-solid interaction potentials. Typical cutoff radii in the range $10-15 \AA$ generate unacceptable errors in the adsorption isotherm if long range corrections are ignored. The large errors incurred by neglecting long range corrections can be concealed by increasing the well depth of the gas-solid interaction. Thus, potential parameters should always be associated with the cutoff radius used for their derivation. Consistency in the cutoff radius is more important than the inclusion or neglect of long range corrections to the energy.

Long range corrections in energy are easily applied to gas-solid interactions using the approximation that the radial distribution function beyond the cutoff radius is unity. Potential parameters derived with long range corrections are practically independent of the cutoff radius.

Support by National Science Foundation Grant CTS0080915 is gratefully acknowleged.

\section{References}

[1] Allen, M. P., and Tildesley, D. J., 1987, Computer Simulation of Liquids (Oxford: Clarendon Press) p. 28.

[2] Rowley, L. A., Nicholson, D., and Parsonage, N. G., 1978, J. comput. Phys., 26, 66.
[3] Goodbody, S. J., Watanabe, K., MacGowan, D., Walton, J. P. R. B., and Quirke, N., 1991, J. chem. Soc. Faraday Trans, 87, 1951.

[4] Smit, B., 1995, J. Phys. Chem., 99, 5597.

[5] Hill, T. L., 1986, An Introduction to Statistical Thermodynamics (New York: Dover) p. 303.

[6] Steele, W. A., 1974, The Interaction of Gases with Solid Surfaces (Oxford: Pergamon Press).

[7] Everett, D. H., and Powl, J. C., 1976, J. chem. Soc. Faraday Trans. 1, 72, 619.

[8] Myers, A. L., Calles, J. A., and Calleja, G., 1997, Adsorption, 3, 107.

[9] Valenzuela, D. P., and Myers, A. L., 1989, Adsorption Equilibrium Data Handbook (Englewood Cliffs, NJ: Prentice-Hall).

[10] Macedonia, M., and Maginn, E., 1999, Molec. Phys., 96, 1375.

[11] Demontis, P., Fois, E. S., Suffritti, G. B., and Quartieri, S., 1990, J. phys. Chem., 94, 4329.

[12] June, R. L., Bell, A. T., and Theodorou, D. N., 1990, J. phys. Chem., 94, 8232.

[13] June, R. L., Bell, A. T., and Theodorou, D. N., 1990, J. phys. Chem., 94, 1508.

[14] Maginn, E. J., Bell, A. T., and Theodorou, D. N., 1995, J. Phys. Chem., 99, 2057.

[15] Clark, L. A., Gupta, A., and Snurr, R. Q., 1998, J. Phys. Chem. B, 102, 6720.

[16] Gupta, A., Clark, L. A., and Snurr, R. Q., 2000, Langmuir, 16, 3910.

[17] Smit, B., and Siepmann, J. I., 1994, J. phys. Chem., 98, 8442.

[18] Nowak, A. K., Den Ouden, C. J. J., Pickett, S. D., Smit, B., and Cheetham, A.K., 1991, J. phys. Chem., 95, 848.

[19] Hufton, J. R., 1991, J. phys. Chem., 95, 8836.

[20] Nicholas, J. B., Trouw, F. R., Mertz, J. E., Iton, L. E., and Hopfinger, A. J., 1993, J. phys. Chem., 97, 4149.

[21] Ermoshin, V. A., and Engel, V., 1999, J. phys. Chem. A, 103, 5116.

[22] Talu, O., and Myers, A. L., 2001, AIChE J., 47, 1160.

[23] Suzuki, S., Takaba, H., Yamaguchi, T., and Nakao, S., 2000, J. phys. Chem. B., 104, 1971.

[24] Olson, D. H., Kokotailo, G. T., Lawton, S. L., and Meier, W. M., 1981, J. phys. Chem., 85, 2238.

[25] Dunne, J. A., Mariwala, R., Rao, M., Sircar, S., Gorte, R. J., and Myers, A. L., 1996, Langmuir, 12, 5888 .

[26] Bull, L.M., and Cheetham, A.K., 1997, Studies Surface Sci. Catal., 105, 471.

[27] Borst, C., 1998, Diploma thesis, Technische Universität München. 